\title{
A Fractal Interaction Model for Winding Paths through Complex Distributions: Application to Soil Drainage Networks
}

\author{
Miguel Ángel Martín, and Miguel Reyes
}

\begin{abstract}
Water interacts with soil through pore channels putting mineral constituents and pollutants into solution. The irregularity of pore boundaries and the heterogeneity of distribution of soil minerals and contaminants are, among others, two factors influencing that interaction and, consequently, the leaching of chemicals and the dispersion of solute throughout the soil.

This paper deals with the interaction of irregular winding dragging paths through soil complex distributions. A mathematical modelling of the interplay between multifractal distributions of mineral/pollutants in soil and fractal pore networks is presented.

A Hölder path is used as a model of soil pore network and a multifractal measure as a model of soil complex distribution, obtaining a mathematical result which shows that the Hölder exponent of the path and the entropy dimension of the distribution may be used to quantify such interplay. Practical interpretation and potential applications of the above result in the context of soil are discussed. Since estimates of the value of both parameters can be obtained from field and laboratory data, hopefully this mathematical modelling might prove useful in the study of solute dispersion processes in soil.
\end{abstract}

Key words: Hölder curves, multifractal distributions, soil drainage networks.

\section{Introduction}

River basins distribute water stored in the soil by releasing it gradually into a complex network that involves a great disparity of length scales from the soil pore channels to the river basin boundaries. Along the way, water interacts with the basin, putting mineral constituent or soil pollutants into solution.

Percolation network theory and fractal models recently have been used for modelling the spreading of solute through porous media during saturated flow. ADLER (1985) considered dispersion in fractal capillary networks, REDNER et al. (1987) studied mechanical dispersion in a self-similar model of a porous medium, and MAzo (1998) studied different aspects of dispersion in fractal media. Fractal curves and networks appear as

Departamento de Matemática Aplicada, Escuela Técnica Superior de Ingenieros Agrónomos, Universidad Politécnica de Madrid, 28040 Madrid, Spain. E-mail: miguelangel.martin@upm.es

Departamento de Matemática Aplicada, Facultad de Informática, Universidad Politécnica de Madrid, Campus de Montegancedo, 28660 Boadilla del Monte, Madrid, Spain. E-mail: mreyes@fi.upm.es 
natural models for irregular geophysical boundaries, river basins or percolation channels (Mandelbrot, 1982; Feder, 1988; Rodríguez-Iturbe and Rinaldo, 1997).

On the other hand, distributions of nutrients and pollutants in soil demonstrate, as many other soil properties, high spatial and temporal variability. KRAvCHENKo et al. (1999) have shown that spatial distributions of soil phosphorus and potassium contents, organic matter contents, calcium and magnesium contents, and cation exchange capacity present highly heterogeneous patterns close to mathematical multifractal distributions. Similarly, mineral deposits have been shown to follow multifractal features (CHENG et al., 1994; Agterberg et al., 1996). These observations imply that the distributions are generally sparse, and denser and rarer regions follow certain scaling regularity. Such heterogeneity should have profound influence on leaching of metals and nutrients, dispersion of solutes throughout the soil, and other transport processes. Both, pore space geometry and the probability that particles or molecules have of being moved from the soil matrix to flow into the pore space, will affect solute transport (PERFECT and SuKOP, 2001). Thus, it is worthwhile to consider modelling the interplay between the complex geometry of pore networks and the heterogeneity of the mass distribution of soil components able to be transported through pore channels during saturated flow.

The objective of this work was to model such mass-geometry interplay using fractal dimension of branched transport pathways and the entropy dimension of spatial distributions of solute concentrations. In section 2 the model is developed and a mathematical result for the model is presented. A precise original mathematical proof of the theoretical result, supporting the value of this modelling, is given. In section 3 the practical interpretation and potential applications of the above result in the context of soil are discussed.

\section{A Fractal Interaction Model for Winding Dragging Paths through Soil Complex Distributions}

Mathematically speaking, a fractal network is a connected (possibly self-intersecting) curve or path of fractal (Hausdorff) dimension $D$. In an abstract setting, this concept directly corresponds with that of Hölder exponent of a continuous path. Since self-similar connected sets of points can be parameterized by means of continuous (Hölder) paths of the same Hausdorff dimension (REMES, 1998; MARTIN AND MATTILA, 2000) we shall model winding pore channels as the image of a Hölder map $f: A \longrightarrow \mathbb{R}^{3}, A \subset \mathbb{R}$, that is, a map verifying $|f(x)-f(y)| \leq c|x-y|^{\alpha}$ for all $x, y \in A$, with $0<\alpha \leq 1$ and $c<\infty$. This model will allow us to obtain exact mathematical results that shall be interpreted later in a practical setting.

On the other hand the entropy dimension is a classical parameter used to quantify heterogeneity of mass distributions (RENYI, 1957) that may be estimated in real distributions by means of multifractal analysis of field data (see next section). 
The above abstract modelling will allow us to give in this section a precise original proof of the following result which shows how the entropy dimension $D_{1}$ of the distribution and the Hölder exponent $\alpha$ of the path, play an important role in measuring the physical interplay between both structures.

Result: Let $\mu$ be a multifractal measure being $D_{1}$ the entropy dimension. Suppose further that $f: A \longrightarrow \mathbb{R}^{n}$ is an $\alpha$-Holder map such that $\gamma=f(\mathbb{R}) \subset S$ and $1 / \alpha<D_{1}$. Then $\mu(\gamma)=0$.

\subsection{Preliminaries}

The mathematical result is crucial for supporting the model. Next we present precise definitions and previous results needed.

Given a finite measure $\mu$ on $\mathbb{R}^{n}$ (or mass distribution), the local dimension (or local Hölder exponent) of $\mu$ at $x \in \mathbb{R}^{n}$ is given by (see e.g., FALCONER, 1997)

$$
\operatorname{dim}_{l o c} \mu(x)=\lim _{r \downarrow 0} \frac{\log \mu(B(x, r))}{\log r}
$$

if this limit exists, where $B(x, r)$ denotes the closed ball $B(x, r)=\left\{y \in \mathbb{R}^{n}\right.$ : $|y-x| \leq r\}, x \in \mathbb{R}^{n}$ and $0<r<\infty$.

For $0 \leq s \leq n$ the $s$-dimensional Hausdorff measure of a set $E \subset \mathbb{R}^{n}$ is

$$
H^{s}(E)=\liminf _{\delta \downarrow 0}\left\{\sum_{i=1}^{\infty} d\left(S_{i}\right)^{s}: E \subset \bigcup_{i=1}^{\infty} S_{i}, d\left(S_{i}\right) \leq \delta\right\} .
$$

In particular, the Hausdorff measure $H^{n}$ is a constant multiple of the Lebesgue measure $\mathcal{L}^{n}$

If $E$ is the support of the measure $\mu$ and $\mathcal{P}=\left\{A_{i}: i=1, \ldots, n\right\}$ is a partition of $E$, the Shannon entropy of $\mu$ with respect to $\mathcal{P}$ is given by (Shannon, 1948)

$$
H_{\mu}(\mathcal{P})=-\sum_{i=1}^{n} \mu\left(A_{i}\right) \log \mu\left(A_{i}\right)
$$

If

$$
H_{\mu}(\varepsilon)=\inf \left\{H_{\mu}(\mathcal{P}): d(\mathcal{P}) \leq \varepsilon\right\}
$$

being $d(\mathcal{P})=\max _{1 \leq i \leq n}\left\{d\left(A_{i}\right)\right\}$, where $d$ stands for the diameter, the entropy dimension of $\mu$ is defined by (RENYI, 1957)

$$
D_{1}=\lim _{\varepsilon \rightarrow 0} \frac{H_{\mu}(\varepsilon)}{-\log \varepsilon}
$$

The Hausdorff dimension of a set $E \subset \mathbb{R}^{n}$ is defined by 


$$
\operatorname{dim}_{H} E=\inf \left\{s: H^{s}(E)=0\right\}=\sup \left\{s: H^{s}(E)=\infty\right\} .
$$

If $m \leq n, 0<\alpha \leq m$, and $A \subset \mathbb{R}^{m}$ we shall denote by $\operatorname{Lip}_{\alpha}\left(A, \mathbb{R}^{n}\right)$ the set of Hölder continuous maps $f: A \longrightarrow \mathbb{R}^{n}$, that is

$$
\operatorname{Lip}_{\alpha}\left(A, \mathbb{R}^{n}\right)=\left\{f: A \longrightarrow \mathbb{R}^{n}: \exists L<\infty \text { with }|f(x)-f(y)| \leq L|x-y|^{\alpha}, \forall x, y \in A\right\} .
$$

The number

$$
L=\sup \left\{\frac{|f(x)-f(y)|}{|x-y|^{\alpha}}: x, y \in A, x \neq y\right\}
$$

is called the Holder constant of $f$.

The next theorem plays an important role in the proof of our results (see MATTLA, 1995).

\subsection{Theorem (Besicovitch's Covering Theorem)}

There are integers $P(n)$ and $Q(n)$ depending only on $n$ with the following properties. Let $A$ be a bounded subset of $\mathbb{R}^{n}$, and let $\mathcal{B}$ be a family of closed balls such that each point of $A$ is the center of some ball of $\mathcal{B}$.

a) There is a finite or countable collection of balls $\left\{B_{i}\right\} \subset \mathcal{B}$ such that they cover $A$ and every point of $\mathbb{R}^{n}$ belongs to at most $P(n)$ balls $B_{i}$, that is,

$$
\chi_{A} \leq \sum_{i} \chi_{B_{i}} \leq P(n)
$$

where $\chi_{A}$ denotes the characteristic function of $A$.

b) There are families $\mathcal{B}_{1}, \ldots, \mathcal{B}_{Q(n)} \subset \mathcal{B}$ covering $A$ such that each $\mathcal{B}_{i}$ is disjoint, that is,

$$
A \subset \bigcup_{i=1}^{Q(n)} \bigcup_{B \in \mathcal{B}_{i}} B \quad \text { and } \quad B \cap B^{\prime}=\emptyset \text { for } \quad B, B^{\prime} \in \mathcal{B}_{i} \quad \text { with } \quad B \neq B^{\prime}
$$

A Hölder map $f: A \longrightarrow \mathbb{R}^{3}, A \subset \mathbb{R}$, gives a parameterization of a fractal path (MattilA, 1995). If the image $f(A)$ is embedded in the support $S$ of a mass distribution, one natural problem is to relate the heterogeneity of the mass distribution, being this measured via the entropy dimension, with the Hölder exponent of the map, in order to create the possibility that the image $f(A)$ can catch a positive amount of mass. The next theorem deals with this problem. It is presented in a general form for distributions in $\mathbb{R}^{n}$.

\subsection{Theorem}

Let $\mu$ be a measure supported on $E \subset \mathbb{R}^{n}$, with entropy dimension $D_{1}$, and $0<s<D_{1}$. Then, for any $(\mathrm{m} / \mathrm{s})$-Holder map $f: A \longrightarrow \mathbb{R}^{n}, A \subset \mathbb{R}^{m}$, we have 


$$
\mu(E \cap f(A))=0 .
$$

Proof. We may assume that $d(E)<1$. Since the entropy dimension is $D_{1}$, then (Young, 1982):

$$
\lim _{r \rightarrow 0} \frac{\log \mu(B(x, r))}{\log r}=D_{1} \quad \text { at } \quad \mu-\text { almost all points } \quad x \in E .
$$

If $s<D_{1}$ is easy to show that

$$
\lim _{r \rightarrow 0} \frac{\mu(B(x, r))}{r^{s}}=0 \quad \text { at } \quad \mu-\text { almost all points } \quad x \in E .
$$

Presume now, contrary to the assertion, that for some $s<D_{1}$ there exists an $(m / s)$-Hölder map $f: A \longrightarrow \mathbb{R}^{n}, A \subset \mathbb{R}^{m}$, with $\mu(E \cap f(A))>0$. Take $\delta>0$ such that $\mu(E \cap f(A))>\delta$. By Egorov's theorem, there is $E_{\delta} \subset E$ such that $\mu\left(E_{\delta}\right)>\mu(E)-\delta$ and

$$
\lim _{r \rightarrow 0} \frac{\mu(B(x, r))}{r^{s}}=0 \quad \text { uniformly on } E_{\delta} \text {. }
$$

Moreover, since $\mu(E \cap f(A))>\delta$, then $\mu\left(E_{\delta} \cap f(A)\right)>0$.

Since Hölder maps can be extended (see VI.2.2 in STEIN, 1970) it may be surmesed that $A$ is open and that $\mathcal{L}^{m}(A)<\infty$.

Since $\mu$-almost all points of $E_{\delta} \cap f(A)$ are $\mu$-density points (see, for example, 2.14 in MAтtLA, 1995), then for $\mu$-almost all $x \in E_{\delta} \cap f(A)$ one has

$$
\lim _{r \rightarrow 0} \frac{\mu\left(E_{\delta} \cap f(A) \cap B(x, r)\right)}{\mu(B(x, r))}=1
$$

and then

$$
\lim _{r \rightarrow 0} \frac{\mu\left(E_{\delta} \cap f(A) \cap B(x, r)\right)}{r^{s}}=0
$$

Let $\varepsilon>0$ arbitrary. Then there is $R>0$ such that

$$
\frac{\mu\left(E_{\delta} \cap f(A) \cap B(x, r)\right)}{r^{s}}<\varepsilon
$$

for $r \leq R$ and for $\mu$-almost all $x \in E_{\delta} \cap f(A)$.

Let consider now the covering of $E_{\delta} \cap f(A)$ formed by balls $B(x, R), x \in E_{\delta}$. Applying Besicovith covering theorem 2.2 , we can get a sequence of balls $\left\{B_{k}\right\}$ verifying (1) such that

$$
E_{\delta} \cap f(A) \subset \bigcup_{k} B_{k}
$$

Moreover, that sequence may be grouped in a finite number of families $\mathcal{B}_{1}, \ldots, \mathcal{B}_{Q(n)}$ being $Q(n)$ a constant depending only on $n$, such that $B \cap B^{\prime}=\emptyset$ for $B, B^{\prime} \in \mathcal{B}_{j}$ with $B \neq B^{\prime}$. 
It follows that there is at least one of these families, say $\mathcal{B}_{j}$, such that

$$
\sum_{B_{i} \in \mathcal{B}_{j}} \mu\left(B_{i} \cap E_{\delta} \cap f(A)\right) \geq \frac{1}{Q(n)} \mu\left(E_{\delta} \cap f(A)\right)
$$

Since the balls $B_{i}$ all have the same radius, the family of balls $\mathcal{B}_{j}$ is finite. Say $\mathcal{B}_{j}=\left\{B_{1}, \ldots, B_{N}\right\}$. Thus one has

$$
N \varepsilon R^{s} \geq \sum_{i=1}^{N} \mu\left(B_{i} \cap E_{\delta} \cap f(A)\right) \geq \frac{1}{Q(n)} \mu\left(E_{\delta} \cap f(A)\right)
$$

and thus

$$
N \geq \frac{\mu\left(E_{\delta} \cap f(A)\right)}{\varepsilon Q(n)} R^{-s}
$$

Let $B_{i}=B\left(x_{i}, R_{\varepsilon}\right)$ with $x_{i} \in E_{\delta} \cap f(A), A_{i}=f^{-1}\left(B_{i}\right)$ and $y_{i} \in A_{i}$ with $f\left(y_{i}\right)=x_{i}$. The Hölder condition implies that

$$
B_{i}^{\prime}=B\left(y_{i}, L^{-s / m} R^{s / m}\right) \subset A_{i} \subset A \quad \text { and } \quad \mathcal{L}^{m}\left(B_{i}^{\prime}\right) \geq c_{m} R^{s} .
$$

Moreover, since the balls $\left\{B_{1}, \ldots, B_{N}\right\}$ are disjoints, the balls $\left\{B_{1}^{\prime}, \ldots, B_{N}^{\prime}\right\}$ are also disjoints. Then

$$
\mathcal{L}^{m}(A) \geq \sum_{i=1}^{N} \mathcal{L}^{m}\left(B_{i}^{\prime}\right) \geq \frac{\mu\left(E_{\delta} \cap f(A)\right)}{\varepsilon Q(n)} R^{-s} c_{m} R^{s}=\frac{\mu\left(E_{\delta} \cap f(A)\right) c_{m}}{\varepsilon Q(n)}
$$

Since $\mu\left(E_{\delta} \cap f(A)\right)>0, c_{m}>0$ and $\varepsilon>0$ is arbitrary, then $\mathcal{L}^{m}(A)=\infty$ which is a contradiction, and the statement follows.

\subsection{Remark}

Notice that rectifiability properties are studied above through coverings formed by balls of equal radius, due to the use of Besicovitch covering theorems instead of Vitali's type that render covering by balls of different sizes. For distributions coming from computer simulation of dynamical systems or else experimental distributions, this seems more convenient: one may not only be interested in limiting properties as rectifiability but also in scaling properties of coverings of controlled size, as obtained in the proof of theorem 2.3.

The entropy dimension thus appears as a degree of accessibility to the mass through continuous paths, giving a measure of the tortuosity needed to catch an important amount of mass.

Invariant measures of dynamical systems produce typical examples of multifractal measures (PESIN, 1996). In the important case of self-similar measures, the result above takes a specific parameterized formulation. Namely, if $\left\{f_{1}, \ldots, f_{N}\right\}$ are contractions in $\mathbb{R}^{n}$ 
and $\left\{p_{1}, \ldots, p_{N}\right\}$ are positive numbers such that $\sum_{i=1}^{N} p_{i}=1$, there is a unique measure verifying

$$
\mu=\sum_{i=1}^{N} p_{i} \mu \circ f_{i}^{-1},
$$

which is called the invariant measure associated to the iterated function system $\left\{f_{1}, \ldots, f_{N}\right.$; $\left.p_{1}, \ldots, p_{N}\right\}$. In the case that the $\left\{f_{1}, \ldots, f_{N}\right\}$ are similarities one has the following result.

\subsection{Corollary}

Let $\left\{f_{1}, \ldots f_{N}\right\}$ be similarities in $\mathbb{R}^{n}$ with contraction ratios $\left\{r_{1}, \ldots, r_{N}\right\},\left\{p_{1}, \ldots, p_{N}\right\}$ positive numbers such that $\sum_{i=1}^{N} p_{i}=1$, and let $\mu$ be the invariant measure with respect to the iterated function system $\left\{f_{1}, \ldots f_{N} ; p_{1}, \ldots, p_{N}\right\}$. Suppose that $\mu\left(f_{i}(E) \cap f_{j}(E)\right)=0$ for $i \neq j$ being $E$ the support of $\mu$. Then if

$$
0<s<\frac{\sum_{i=1}^{N} p_{i} \log p_{i}}{\sum_{i=1}^{N} p_{i} \log r_{i}}
$$

for any $(m / s)$-Hölder map $f: A \longrightarrow \mathbb{R}^{n}, A \subset \mathbb{R}^{m}$, we have $\mu(E \cap f(A))=0$.

Proof. It is a direct consequence of the fact that the entropy dimension of self-similar measures is given by the formula (DELIU et al., 1991):

$$
D_{1}=\frac{\sum_{i=1}^{N} p_{i} \log p_{i}}{\sum_{i=1}^{N} p_{i} \log r_{i}}
$$

\section{Applications to Soil Drainage Networks}

Field data corresponding to soil properties can be collected in one, two or three spatial dimensions. The characteristics (i.e., shape, size and connectivity) of pore networks are often studied by two-dimensional image analysis of thin sections. Similarly, information on the spatial variability of soil properties is usually collected at different sites (points) located along a transect or over a given area. Thus, fractal modelling of the boundaries of pore channels and capillaries can be made by means of fractal curves and networks. Analogously, mineral or contaminant concentrations in soil may be represented by means of a mass distribution. We develop in this section a mass-geometric fractal modelling of the interplay between pore space geometry and the mass distribution of nutrients and contaminants. 


\subsection{Fractal Pore Channel Networks}

Soil is formed by an intricate arrangement of solid particles and voids (pores) with connecting pore channels through which fluid flow and solute transport take place. A number of different approaches have been used to model this situation (JURY and FLÜER, 1992), including those which apply fractal and percolation models (SAHIMI, 1993; AdLER, 1985; among others). The fractal nature of pore boundaries within a range of scales has been demonstrated. The concept of tortuosity applied to pore channels, widely used in soil sciences, has a precise meaning in terms of the scaling behavior of the length of pores. Although a wide variety of models has been used to describe pore geometry (PERFECT and Sukop, 2001) an ideal pore channel may be modelled by a fractal curve.

In order to quantify pore channel tortuosity the boundary fractal dimension $D$ is used which is defined by means of the scaling equation (KAMPICHCHLER and HAUSER, 1993; ANDERSON et al., 1998; PACHEPSKY et al., 1996):

$$
L(\varepsilon) \approx L_{1} \varepsilon^{1-D}
$$

where $L(\varepsilon)$ is the measured length using a yardstick of normalized length $\varepsilon$, and $L_{1}$ is the measured length when $\varepsilon$ is equal to unity.

Different values for the fractal dimension of pore boundaries ranging from 1.06 to 1.51 have been found. Also the effect of management practices on such values has been studied (PACHEPSKY et al., 1996).

\subsection{Parameterizing Heterogeneity of Soil Distributions}

The distribution of soil mineral components and pollutants also show a high spatial variability. Since water interacts with soil mineral constituents and pollutants through soil pore channels, the spatial variability of their concentrations is a factor that should be taken into account in the study of solute dispersion processes. Thus, the geometry of the distribution of some soil minerals or contaminants is a crucial feature to determine the accessibility of pore channels to disperse soil componentes. For studying those distributions one may consider a measure or distribution that assigns to every region $E$ the quantity $\mu(E)$ of a certain component located in that region. Typically $\mu(E)$ depends on the location of that region of the medium and varies widely with respect to the volume of $E$, having the main features of multifractal measures. This implies that, being sparse within the solid matrix, there exists denser and rarer regions following certain scaling regularity. In order to characterize the complexity of the spatial distribution, multifractal analysis may be used to estimate the Renyi spectrum of dimensions which include the entropy dimension as a significant dimension (see Everstz and Mandelbrot, 1992).

Let $S$ be the support of a distribution $\mu$ (for methodological reasons we use a twodimensional model here). Let $\mathcal{P}=\left\{R_{i}\right\}_{i=1}^{N}$ be a collection of squares of side length $\varepsilon$ (see Fig. 1) that represent a partition of $S$. 


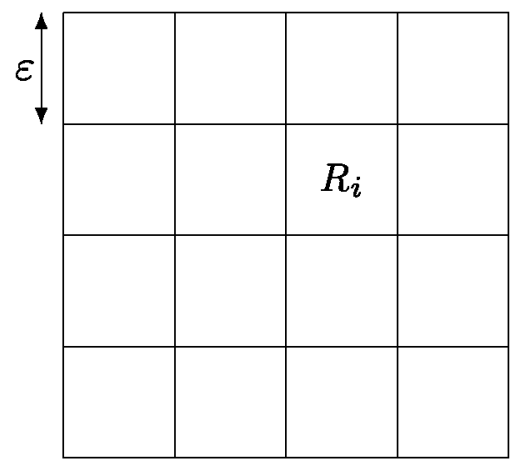

Figure 1

The Renyi dimensions are defined as

$$
D_{q}=\frac{1}{q-1} \lim _{\varepsilon \rightarrow 0} \frac{\log \sum_{i=1}^{N(\varepsilon)} \mu_{i}(\varepsilon)^{q}}{\log \varepsilon},
$$

for $q \neq 1$, being $D_{1}$ the entropy dimension computed by

$$
D_{1}=\lim _{\varepsilon \rightarrow 0} \frac{\sum_{i=1}^{N(\varepsilon)} \mu_{i}(\varepsilon) \log \mu_{i}(\varepsilon)}{\log \varepsilon} .
$$

The dimension $D_{0}$ is called the capacity dimension which agrees with the fractal dimension of the support $S$.

When $D_{q}$ is a decreasing function of $q, \mu$ is called a multifractal distribution.

\subsection{Application to Soil Drainage}

The theoretical result of section 2 may be interpreted in the practical context of soil. It suggests to use the exponent $D_{1}-D$ as an indicator that might reflect the likelihood of leaching or dispersion of soil chemicals and minerals, based solely on the physical interplay between the pore boundary and chemical molecules. The greater the index is the higher is the probability of chemical molecules to be dispersed into the water pore channel. This probability would diminish when the difference $D_{1}-D$ approaches zero and increases when it becomes negative. The greater the entropy dimension is, the more tortuosity for the channel is needed, and thus this parameter may be used as a measure of the risk of mineral loss by illuviation.

In the case of soil contaminants it would measure the risk of exporting pollution to the surrounding areas. Particle size soil distributions have been shown to obey fractal scaling laws (Turcotte, 1986; Tyler and WheAtcraft, 1990) and the powerscaling exponent has been related with tortuosity (TYLER and WHEATCRAFT, 1989). 
Thus the knowledge of such exponent or other entropy-like quantities (MARTín et al., 2001) characterizing texture, together with the entropy dimension of certain distributions of soil mineral, provide valuable information that may be used for the diagnosis of soils and eventually may be of help in implementing adequate policies. In this sense, the potential application might be wide and have a real value in a practical setting. It is well known that parameters and meaningful indexes, such as those above, are strongly demanded by soil scientists (DORAN and PARKIN, 1994).

\subsection{A Case Study}

Soil samples corresponding to an agricultural field (vineyard) located in Central Spain have been collected. A total number of 256 sampling points in an square lattice was considered, being the distance between two neighboring points equal to 20 meters. At any point of the lattice a sample at $25 \mathrm{~cm}$. depth was taken and potassium, phosphorous and organic matter contents were obtained by laboratory standard techniques. This produces data sets $\left\{\mu_{i}: i=1,2, \ldots, 256\right\}$ corresponding to the respective potassium/phosphorous and organic matter contents.

The probability measure or mass distribution $\mu$ is constructed, assigning to any subsquare $R \subset S$ a measure or mass

$$
\mu(R)=\frac{\sum_{x_{i} \in R} \mu_{i}}{\sum_{x_{i}} \mu_{i}}
$$

At every $\mu_{i}(\varepsilon)$ for $i=1,2, \ldots, N(\varepsilon)$ is computed.

The multifractal dimensions $D_{q}$ are estimated by a least-square fitting of the corresponding scalings endowed in equation (1), for $\varepsilon$ ranging from $\varepsilon=16$ to $\varepsilon=1$, and $q$ ranging from $q=-10$ to $q=10$ with a lag of 0.5 . The multifractal dimensions $D_{q}$ are plotted against $q$ in Figures 2 and 3 .

Coefficients of determination $\left(R^{2}\right)$ in those fittings and $D_{q}$ values range from 0.993 to 0.999 for $q$ values ranging from $q=-10$ to $q=10$.

The estimated values of the entropy dimensions of potassium and phosphorous distributions were 1.973 and 1.964 respectively which means that both distributions have followed quite similar heterogeneity patterns. It would mean that both minerals have a very similar probability to be in contact with soil water during saturated flow.

Taking into account the increase of the pore boundary dimension value after tillage and other management practices (PACHEPSKY et al., 1996) the exponent $D_{1}-D$ would be affected and consequently the probability of leaching of different minerals by the effect of watering or rain events. It follows that the use of parameters proposed here might be useful to assess the planning of management practices in agricultural fields. 


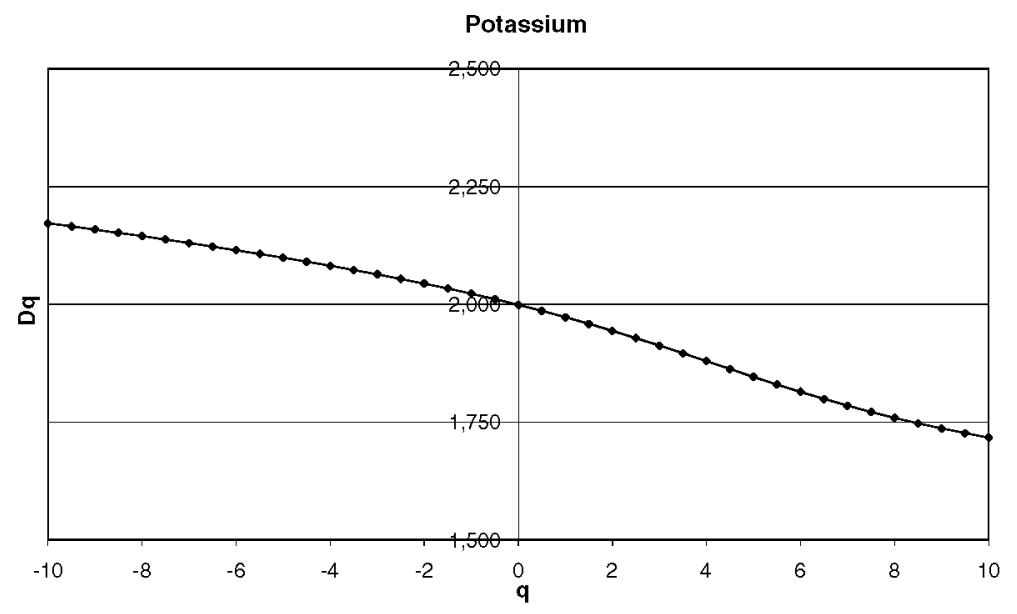

Figure 2

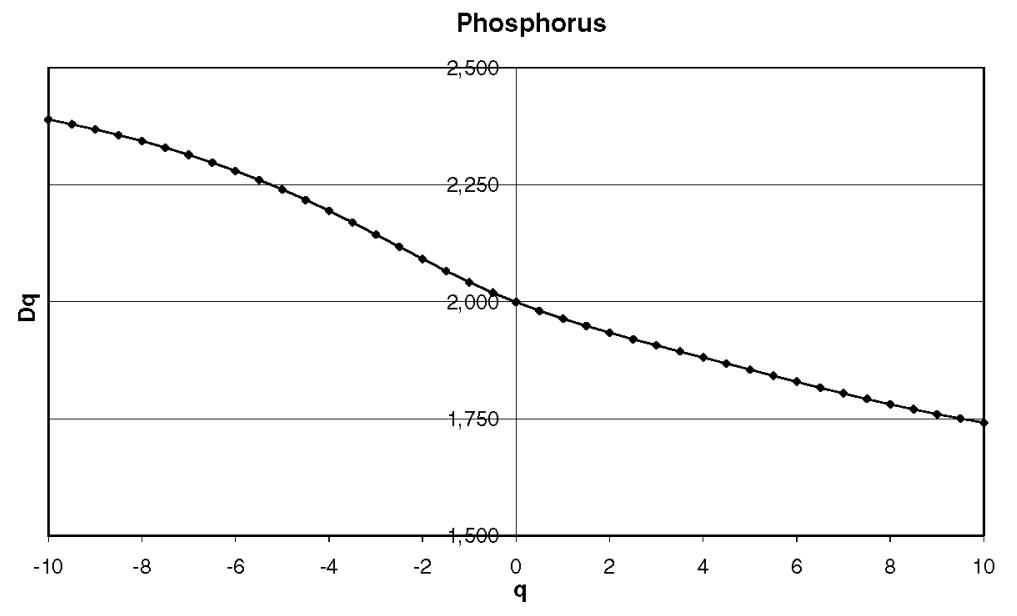

Figure 3

\section{Conclusions}

Physical and chemical heterogeneities coexist together and interplay in soil. Under a modelling of this interplay, the boundary of pore space may be characterized by the fractal dimension $D$ and the heterogeneity of soil minerals or contaminants chemical may be characterized by the entropy dimension $D_{1}$. A mathematical result is precisely derived and interpreted in a practical context. If $D \ll D_{1}$ the pore network has only a small 
probability of catching the chemical mass spread in a heterogeneous multifractal manner. This probability would diminish when the difference $D_{1}-D$ approaches zero and increases when it becomes negative. Although this is a simple and schematic modelling of a considerably more complex situation, this result relates to factors that influence solute dispersion and appear unconnected in former studies. Since estimates of the value of both parameters can be obtained from laboratory and field data, hopefully this mathematical modelling might be useful in future studies

\section{Acknowledgments}

This work has partially been supported by Plan Nacional de Investigación Científica, Desarrollo e Innovación Tecnológica (I+D+I) (Spain), Ref.: AGL2007-62648, and by DGUI (Comunidad de Madrid) - UPM (Technical University of Madrid), Ref.: M0700204135.

\section{REFERENCES}

ADLER, P.M. (1985), Transport processes in fractals. III. Taylor dispersion in two examples of fractal capillary networks, Int. J. Multiphase Flow 11, 241-254.

Agterberg, F.P., Cheng, O., Brown, A., and Good, D. (1996), Multifractal modelling of fractures in the Lac Du Bonnet batholith, Manitoba, Comput. Geosci. 22, 497-507.

Anderson, A.N., McBratney, A.D., and Crawford, J.W. (1998), Application of fractals to soil studies, Advances in Agron. 63, 1-76.

Cheng, O., Agterberg, F.P., and Ballantrne, S.B. (1994), The separation of geochemical anomalies from background by fractal models, J. Geochem. Explor. 51, 109-130.

Deliu, A., Geronmo, J.S., Shonkwiler, R., and Hardin, D. (1991), Dimensions associated with recurrent selfsimilar sets, Math. Proc. Cambridge Philos. Soc. 110, 327-336.

Doran, J.W. and PARKIN, T.B., Defining and assessing soil quality. In Defining Soil Quality for a Sustainable Environment (eds. Doran, J.W., Coleman, D.C., Bezdicek, D.F., and Stewart, B.A.). (SSSA Special Publication number 35, Madison, Wisconsin, USA. Soil Science Society of America 1994) pp. 3-22.

Evertsz, C.F.G. and MAndelbrot, B.B., Multifractal measures. In Chaos and Fractals (eds. Peitgen, H.O., Jürgens, H. and Saupe, D.) (Springer Verlag, New York 1992) pp. 921-953.

FALCONER, K.J., Techniques in Fractal Geometry (John Wiley \& Sons, Chichester 1997).

Feder, J., Fractals (Plenum Press, New York 1988).

JURY, W.A. and FlöhlER, H. (1992), Transport of chemicals through soil: Mechanisms, models and field applications, Adv. Agron. 47, 141-201.

KAMPICHChler, C. and HAuser, M. (1993), Roughness of soil pore surface and its effect on available habitat space of microarthropods, Geoderma 56, 223-232.

Kravchenko, A.N., Boast, C.W., and Bullock, D.G. (1999), Multifractal analysis of soil spatial variability, Agron. J. 91, 1033-1041.

Mandelbrot, B.B., Fractal Geometry of Nature (Freeman, New York 1982).

Martín, M.A. and MATtLA, P. (2000), On the parameterization of self-similar and other fractal sets, Proc. Am. Math. Soc. 128, 2641-2648.

MARTín, M.A., REY, J.M. and TAGUAS, F.J. (2001), An entropy-based parameterization of soil texture via fractal modelling of particle-size distribution, Proc. R. Soc. London Ser. A 457, 937-948.

MatTILA, P., Geometry of Sets and Measures in Euclidean Spaces (Cambridge University Press, Cambridge 1995). 
Mazo, R.M. (1998), Taylor dispersion on a fractal, Acta Phys.Polon. B 29, 1539-1549.

Pachepsky, Y., Yakovchenko, V., Rabenhost, M.C., Pooley, C. and Sikora, L.J. (1996), Fractal parameters of pore surface as derived from micromorphological data: effect of long-term management practices, Geoderma 74, 305-319.

Perfect, E. and Sukor, M.C., Models relating solute dispersion to pore space geometry in satured media: A review. In Physical and Chemical Processes of Water and Solute Transport / Retention in Soil (eds. Selim, H.M. and Sparks, D.L.). (Special Publ. 56, Soil Sci. Soc. Am., Madison WI 2001) pp. 77-146.

PesIn, Y., Dimension Theory in Dynamical Systems (Chicago University Press, Chicago 1996).

ReDner, S., Koplik, J. and WILKInson, D. (1987), Hydrodynamic dispersion in a self-similar geometry, J. Phys. A 20, 1543-1555.

Remes, M. (1998), Holder parameterization of self-similar sets, Ann. Acad. Sci. Fenn. Ser A I Math, Dissertations 112 .

RENYI, A., Dimension, entropy and information. In Statistical Decision Functions and Random Processes (Trans. 2nd Prague Conf. on Information Theory, 1957) pp. 545-556.

Rodríguez-Iturbe, I. and Rinaldo, A., Fractal River Basins, Chance and Self-organization (Cambridge University Press, Cambridge 1997).

SAHIM, M. (1993), Flow phenomena in rocks: From continuum models to fractals, percolation, cellular automata, and simulated annealing, Modern Physics 65, 1393-1534.

Shannon, C.E. (1948), A mathematical theory of communication I, Bell Syst. Tech. J. 27, 379-423.

STEIN, E.M., Singular Integrals and Differentiability Properties of Functions (Princeton University Press, Princeton 1970).

TurcotTe, D.L. (1986), Fractals and fragmentation, J. Geophys. Res. 91, 1921-1926.

Tyler, S.W. and Wheatcraft, S.W. (1989), Application of fractal mathematics to soil water retention estimation, Soil Sci. Soc. Am. J. 53, 987-996.

Truer, S.W. and Wheatcraft, S.W. (1990), Fractal processes in soil water retention, Water Resour. Res. 26 , 1047-1054.

Young (1982), Dimension, entropy and Lyapunov exponents, Ergodic Theory Dynamical Systems 2, $109-124$. 